\title{
Microarrays for the scalable production of metabolically relevant tumour spheroids: a tool for modulating chemosensitivity traits $\dagger$
}

\author{
Heike Hardelauf, $\dagger^{a}$ Jean-Philippe Frimat, ${ }^{a}$ Joanna D. Stewart, ${ }^{b}$ Wiebke Schormann, ${ }^{b}$ Ya-Yu Chiang, ${ }^{a}$ \\ Peter Lampen, ${ }^{a}$ Joachim Franzke, ${ }^{a}$ Jan G. Hengstler, ${ }^{b}$ Cristina Cadenas, ${ }^{a}$ Leoni A. Kunz-Schughart ${ }^{c}$ \\ and Jonathan West $* a$
}

\author{
Received 10th June 2010, Accepted 12th October 2010 \\ DOI: 10.1039/c0lc00089b
}

We report the use of thin film poly(dimethylsiloxane) (PDMS) prints for the arrayed mass production of highly uniform 3-D human HT29 colon carcinoma spheroids. The spheroids have an organotypic density and, as determined by 3-axis imaging, were genuinely spherical. Critically, the array density impacts growth kinetics and can be tuned to produce spheroids ranging in diameter from 200 to $550 \mu \mathrm{m}$. The diffusive limit of competition for media occurred with a pitch of $\geq 1250 \mu \mathrm{m}$ and was used for the optimal array-based culture of large, viable spheroids. During sustained culture mass transfer gradients surrounding and within the spheroids are established, and lead to growth cessation, altered expression patterns and the formation of a central secondary necrosis. These features reflect the microenvironment of avascularised tumours, making the array format well suited for the production of model tumours with defined sizes and thus defined spatio-temporal pathophysiological gradients.

Experimental windows, before and after the onset of hypoxia, were identified and used with an enzyme activity-based viability assay to measure the chemosensitivity towards irinotecan. Compared to monolayer cultures, a marked reduction in the drug efficacy towards the different spheroid culture states was observed and attributed to cell cycle arrest, the 3-D character, scale and/or hypoxia factors. In summary, spheroid culture using the array format has great potential to support drug discovery and development, as well as tumour biology research.

\section{Introduction}

Monolayer cell cultures are traditionally used as in vitro models to investigate the cancer process and identify effective anti-tumour therapies. These planar models do not recapitulate the tissue architecture, cell-cell and cell-matrix interactions, and mass transfer barriers and associated metabolic gradients of avascular tumour nodules, micrometastases or intervascular tumour regions. This limits their predictive value, leading to the potential failure of therapies during in vivo trials, or the failure of potentially effective therapies to progress to further testing phases. As an alternative, the multicellular tumor spheroid model has emerged as a powerful spherical tissue model with authentic cell-cell interactions and complete radial symmetry for the establishment of uniform mass transfer gradients typical of the tumour niche. ${ }^{1,2}$

\footnotetext{
${ }^{a}$ Leibniz-Institut für Analytische Wissenschaften-ISAS-e.V., Otto-Hahn-Str.6b, 44227 Dortmund, Germany.E-mail: west@isas.de ${ }^{b}$ Leibniz Research Centre for Working Environment and Human Factors at the University of Dortmund (IfADo), Ardeystr. 67, 44139 Dortmund, Germany

'OncoRay-Center for Radiation Research in Oncology, Tumor Pathophysiology, TU Dresden, Fetscherstraße 74, 01307 Dresden, Germany

$\dagger$ Electronic supplementary information (ESI) available: Thin film PDMS printing (Fig. S1), assembly and growth video, integrin and E-cadherin gene expression (Fig. S2), BT474 and NCI-H1792 spheroid arrays (Fig. S3), volumetric growth (Fig. S4), necrosis onset (Fig. S5) and details of the chemosensitivity experiment (Table S1). See DOI $10.1039 / \mathrm{c} 01 \mathrm{c} 00089 \mathrm{~b}$

\$ née Menne.
}

Multicellular tumour spheroids are traditionally produced by a variety of methods which share the common feature of resisting cell-surface interactions to promote cell-cell coupling and the aggregation of dense cellular assemblies. One strategy involves the continuous agitation of the cell suspension within spinner flasks, roller tubes, gyratory shakers or rotating wall vessels. ${ }^{1,3}$ These methods are suitable for large scale production, but are lengthy and produce heterogeneously sized spheroids. Alternatively, for smaller scale operations, the aggregation process can be achieved by sedimentation onto concave and cell adhesion resistant surfaces, such as microtitre plate wells coated with agarose, ${ }^{4}$ poly-hydroxyethyl methacrylate (poly-HEMA),${ }^{5}$ or a droplet's air-liquid interface. ${ }^{6-8}$ These methods compartmentalize the aggregation of individual spheroids and result in the formation of homogeneously sized spheroids. However, these methods suffer the drawbacks of being tedious, manually intensive and with a limited scope for mass production. To drive the widespread implementation of the spheroid model in routine anti-cancer therapy testing programs new automated methods for the scalable production of tumour spheroids with uniform characteristics are required. ${ }^{2}$

Tissue engineering approaches have been used to tackle the problem by encapsulating the aggregation process within scaffold pockets formed by templating monodisperse microparticles $^{9,10}$ or droplets prepared within a two-phase microfluidic reactor. ${ }^{11}$ Microfluidics has also received great interest as a means to provide continuous perfusion during compartmentalized spheroid assembly. Devices are fabricated from poly(dimethylsiloxane) (PDMS) for ease of replication and its cell 
adhesion resistance properties. ${ }^{12,13}$ To avoid disrupting aggregation a bilayer system incorporating a semi-porous membrane can be used to vertically interface the culture chamber with a perfusion channel, ${ }^{14-18}$ or rows of micropillars can be used to interface a central culture chamber with neighbouring perfusion channels. ${ }^{19,20}$ To similar effect, hydrodynamic traps can also be used to shelter cellular aggregates during continuous perfusion. ${ }^{21}$

Microwell arrays can also be used to cage the assembly process during stationary culture. Here, the cells settle from suspension into discrete populations of equivalent number for the automated culture of uniformly sized spheroids. The microwell format can be highly parallelized for the high density culture of spheroids numbering in their thousands. Microwell arrays have been fabricated in poly(methyl methacrylate) (PMMA) by UVLIGA, ${ }^{22}$ or in PDMS using anisotropically etched silicon ${ }^{23}$ or microstructured SU-8 moulds. ${ }^{24-26}$ Poly(ethylene glycol) (PEG) is another preferred material which enables spheroid formation by resisting cell adhesion, with microwell arrays either made by embossing a PEG material layer ${ }^{27}$ or by the self-assembly of thiolated PEG molecules onto platinum-coated PMMA microwells. ${ }^{28}$ Collagen $^{29,30}$ or Arg-Gly-Asp peptide ${ }^{31}$ adhesion islands have also been microcontact printed $(\mu \mathrm{CP})$ onto the base of the microwells to centre spheroid growth. Importantly, adhesion patterns alone can be used to direct the centre of cell assembly and partition growth for the parallel culture of spheroids. Here, the physical walls of microfluidic and microwell systems are replaced with a biologically inert background such as PEG brushes or hydrogels. ${ }^{32-36}$ This planar compartmentalization strategy enables unconstrained growth with improved mass transfer for nutrient supply and waste removal.

The vast majority of microtechnology-based spheroid production methods have focused on the development of 3-D liver tissue models for the maintenance of hepatocyte-specific biochemical and metabolic functions or for the controlled and synchronized differentiation of stem cells into embryoid bodies. In this paper we instead report the use of thin film PDMS micropatterning for the controlled culture of uniform and metabolically relevant tumour spheroids and demonstrate the value of this approach for cancer research and drug discovery applications.

\section{Materials and methods}

\section{Microarray-based spheroid culture}

Throughout this study hexagonally arrayed cell adhesion islands with a diameter of $150 \mu \mathrm{m}$ were patterned on glass substrates across an area of $20 \times 20 \mathrm{~mm}$. Arrays with different adhesion island pitches and different numbers of adhesion islands were used in this study: $400 \mu \mathrm{m}(\times 2411), 450 \mu \mathrm{m}$ (25 sub-arrays totalling $1675), 700 \mu \mathrm{m}(\times 739), 1000 \mu \mathrm{m}(\times 409), 1250 \mu \mathrm{m}(\times 263), 1500 \mu \mathrm{m}$ $(\times 149)$ and $2000 \mu \mathrm{m}(\times 104)$. As illustrated in ESI Fig. S1†, cell patterning was achieved by thin film PDMS microcontact printing as described previously by Frimat and co-workers. ${ }^{13}$ Briefly, SU-8 moulds were prepared by standard photolithographic methods and used to cast PDMS (Sylgard ${ }^{\circledR}$ 184, Dow Corning) stamps at $70{ }^{\circ} \mathrm{C}$ for 20 minutes. The SU-8 mould incorporates protruding circular structures for the fabrication of PDMS stamps with recessed features. The stamps were inked by contact transfer for $\sim 10 \mathrm{~s}$ with a liquid PDMS deposit prepared by spin coating a $500 \mu \mathrm{L}$ volume of PDMS prepolymer and curing agent dissolved in chloroform $(1: 10, \mathrm{w} / \mathrm{w})$ for $30 \mathrm{~s}$ at $6000 \mathrm{rpm}$. Printing was also achieved by contact transfer for $\sim 10 \mathrm{~s}$. A sacrificial print was used to remove excess liquid PDMS for subsequent precision printing on glass substrates. Thermal curing at $70{ }^{\circ} \mathrm{C}$ for 10 minutes was used to produce a stable thin film PDMS perforation pattern exposing areas of the underlying substrate. The glass surface bordering the pattern was also passivated with PDMS to restrict cells to the adhesion islands alone.

Human colon carcinoma cells (HT29), BT474 breast carcinoma and NCI-H1792 lung carcinoma cell lines were purchased from DSMZ (Germany) and ATCC (USA). Cells were cultured in Dulbecco's modified Eagle media (DMEM) supplemented with $10 \%(\mathrm{v} / \mathrm{v})$ foetal bovine serum (or $10 \%(\mathrm{v} / \mathrm{v})$ foetal bovine serum gold (PAA, Germany) for the HT29 cell line), $1 \%$ (v/v) Glutamax, and $1 \%(\mathrm{v} / \mathrm{v})$ penicillin and streptomycin (Sarstedt AG \& Co., Germany). Cells were cultured at $37^{\circ} \mathrm{C}$ in a humidified atmosphere with $6 \% \mathrm{CO}_{2}$, and harvested using trypsin/EDTA once 80 $90 \%$ confluency was attained. The arrays were placed within hydrophobic, bacteriological grade Petri dishes $(\varnothing=55 \mathrm{~mm})$ and seeded with a $1 \mathrm{~mL}$ media suspension containing $2 \times 10^{5}$ cells and incubated for 3 days prior to gentle washing and media exchange to remove non-adherent cells. Following cell patterning a $6 \mathrm{~mL}$ media volume was periodically exchanged every $2-3$ days. For offchip analysis spheroids were harvested by pipetting.

\section{Physical characterisation}

The spheroids were documented using an inverted microscope (IX71, Olympus). High resolution spheroid array images were obtained using an environmental SEM (Quantam200F, FEI) operating at $90 \mathrm{~Pa}$. SEM imaging required fixation. The arrays were incubated twice in $1 \times$ PBS for 5 minutes and then fixed for 24 hours in SAV neutral buffered $4 \%$ formaldehyde (Liquid Production, Germany), followed by a final $1 \times$ PBS wash for 5 minutes and air drying for a further 24 hours. The spheroid arrays were coated with gold for electron imaging. Spheroid morphology was determined by 3 -axis imaging, with circularity measurements using ImageJ (NIH). Spheroids were first embedded in a hydrated agarose environment by insertion into molten $2 \%(\mathrm{w} / \mathrm{v})$ agarose, followed by cooling for gelation. A razor blade was used to cut agarose cubes for straightforward 3axis positioning. The spherical nature of the spheroids enables the tissue density to be estimated from the sedimentation velocity (assuming steady-state motion). Spheroid sedimentation velocities within a $1 \times$ PBS column were recorded and a derivation of the Stoke's equation was used to calculate the density:

$$
\rho_{\mathrm{s}}=\rho_{\mathrm{f}}+\left(\frac{9 \eta v_{\mathrm{s}}}{2 r_{\mathrm{s}}{ }^{2} g}\right)
$$

where $\rho_{\mathrm{s}}$ is the spheroid density, $\rho_{\mathrm{f}}$ the fluid density, $\eta$ the fluid viscosity $\left(1.002 \times 10^{-3} \mathrm{~kg} \mathrm{~m}^{-1} \mathrm{~s}^{-1}\right)$, $v_{\mathrm{s}}$ the sedimentation velocity, $r_{\mathrm{s}}$ the spheroid radius and $g$ gravity $\left(9.8 \mathrm{~m} \mathrm{~s}^{-2}\right)$. For each growth condition, five 3-axis measurements were taken. The number of cells per spheroid involved pooling 30 spheroids in triplicate. The supernatant was removed and the spheroids were treated with trypsin for 30 minutes at $37^{\circ} \mathrm{C}$ with vortexing every 5 minutes. A $100 \mu \mathrm{L}$ volume of this single cell suspension was diluted in $10 \mathrm{~mL}$ 
Casyton for cell counting with a Casy ${ }^{\circledR}$ instrument (Innovatis AG, Germany).

The Gompertz equation can be used to accurately model the volumetric growth of avascularised spherical tissues. ${ }^{37-39}$ The spheroid volume at a given time, $V_{(\mathrm{t})}$, is given by:

$$
V_{(t)}=V_{(0)} \times \exp ^{\alpha / \beta\left(1-\exp ^{\beta_{t}}\right)}
$$

where $V_{(0)}$ is the initial spheroid volume, $\alpha$ is the growth regression rate and $\beta$ is a growth retardation constant. The Gompertz equation was used to evaluate the growth behaviour of spheroids on microarrays with pitches ranging from 400 to $2000 \mu \mathrm{m}$. The spheroid volume, $V_{\mathrm{s}}$, was calculated from the diameter using a capped spherical model:

$$
V_{\mathrm{s}}=\left(4 / 3 \pi r^{3}\right)-\left(\pi h\left(3 a^{2}-h^{2}\right) / 6\right)
$$

where $r$ is the radius of a sphere (spheroid), $a$ the radius of the cap (adhesion island) and $h$ the height of the cap.

\section{Biological characterisation}

Gene expression analysis. For reverse transcription quantitative polymerase chain reaction (RT-qPCR) analysis, RNA was isolated from spheroid and monolayer cultures using QIAzol Lysis Reagent (Qiagen) following the manufacturer's protocol. Two micrograms of total RNA were reverse-transcribed using random primers and the High Capacity cDNA Reverse Transcription Kit (Applied Biosystems). RT-qPCR was undertaken with an ABI Prism 7700 (Applied Biosystems) and the QuantiTect SYBR Green PCR Kit (Qiagen). QuantiTect primer assays (Qiagen) were used for the amplification of all gene expression targets: actin (QT01680476); $\alpha 5 \beta 1$-integrin (QT00068124); E-cadherin (QT00080143); PCNA (QT00024633); cyclin D (QT004925285); p21 (QT00062090); and VEGF-A (QT01682072). Amplification involved a 15 minute hot start at $95^{\circ} \mathrm{C}$, followed by 40 cycles of denaturation at $94{ }^{\circ} \mathrm{C}$ for $15 \mathrm{~s}$, annealing at $60{ }^{\circ} \mathrm{C}$ for $30 \mathrm{~s}$, extension at $72{ }^{\circ} \mathrm{C}$ for $35 \mathrm{~s}$, and finishing with ramping from $55^{\circ} \mathrm{C}$ to $95{ }^{\circ} \mathrm{C}$ to generate melting curves. Data were analysed using the $2^{-\Delta \Delta \mathrm{Ct}}$ method $^{40}$ with actin used as the housekeeping gene and the untreated monolayer cultures used as the calibrator. Each condition was undertaken in triplicate, with each sample measured in triplicate.

Section staining. For imaging tissue sections, spheroids were embedded in $1 \%$ agarose, fixed in $4 \%$ buffered formaldehyde overnight at $4{ }^{\circ} \mathrm{C}$, and subsequently embedded in paraffin for cutting into $4 \mu \mathrm{m}$ thick sections using a microtome (Microm HM450). Immediately prior to staining, the paraffin was removed by rinsing in Rotihistol $(4 \times 5$ minutes), followed by hydration using an ethanol dilution series $(100 \%, 90 \%, 70 \%, 50 \%$ and $30 \%$, each 5 minutes) with final rinsing in water. Spheroid sections were stained with Mayer's haematoxylin (Merck) for 5 minutes followed by a water rinse and then with $1 \%$ eosin (Merck) for 3 minutes followed by a second water rinse. Sections were then dehydrated in isopropanol and mounted with Entellen ${ }^{\circledR}$ (Merck) for microscopy imaging.

Proliferating cells were identified using a 5-bromo-2-deoxyuridine $(\mathrm{BrdU})$ incorporation assay, involving incubation in $10 \mu \mathrm{M}$ BrdU for 6 hours, followed by formaldehyde fixation. For
BrdU and HIF-1 $\alpha$ immunostaining, antigens were made accessible by placing the slides in a citrate buffer $(0.01 \mathrm{M}, \mathrm{pH} 6.0)$ and heating in a microwave $(2 \times 7$ minutes $)$. Following cooling, sections from spheroids incubated with BrdU were further treated for 10 minutes with $2 \mathrm{~N} \mathrm{HCl}$. All slides were then rinsed twice in $1 \times$ PBS and further prepared in a humidity chamber. Non-specific binding was blocked with $3 \%$ BSA/PBS $/ 0.1 \%$ Tween 20 for 1 hour followed by the addition of the primary antibodies which were directed against BrdU (rat anti-BrdU, Serotec, $1: 25$ in $0.3 \%$ BSA/ $1 \times$ PBS $/ 0.1 \%$ Tween 20 ) or HIF- $1 \alpha$ (mouse anti-HIF-1 $\alpha$, Novus Biologicals, $1: 25$ in $0.3 \%$ BSA $/ 1 \times$ PBS $/ 0.1 \%$ Tween 20$)$ and incubation for 1 hour, followed by $1 \times$ PBS washing $(3 \times 5$ minutes $)$. Slides were then incubated with Cy2-conjugated rat or mouse secondary antibodies (Dianova, Hamburg, $1: 100$ in $0.3 \% \mathrm{BSA} / 1 \times \mathrm{PBS} / 0.1 \%$ Tween 20 ) for 1 hour and washed with $1 \times \operatorname{PBS}(3 \times 5$ minutes $)$. The nuclei were stained for 5 minutes with 4',6-diamidino-2-phenylindole (DAPI, Invitrogen) at room temperature. Slides were mounted with Mowiol solution and a laser confocal scanning microscope (Fluoview 1000, Olympus) was used for fluorescent imaging.

Irinotecan dose-response analysis. A dose-response experiment using the anti-cancer drug irinotecan was undertaken to identify the altered chemosensitivity states of tumour spheroids. Arrays with pitches of 400 and $1500 \mu \mathrm{m}$ were used to culture spheroids with irinotecan exposure for 3 days at concentrations ranging from $3.16 \mu \mathrm{M}$ to $1000 \mu \mathrm{M}$ during pre-hypoxic and hypoxic phases of culture. Monolayer cultures were used as experimental controls. Microtitre plate wells were each seeded with $6 \times 10^{2}$ cells, cultured for 4 days at $37{ }^{\circ} \mathrm{C}$ in a $6 \% \mathrm{CO}_{2}$ atmosphere and then exposed to irinotecan at concentrations ranging from $1 \mu \mathrm{M}$ to $3.16 \mathrm{mM}$ for a further 3 days. The acid phosphatase assay was used to measure the inhibitory effects of irinotecan on cell viability. ${ }^{41,42}$ Following exposure the spheroids were washed twice with $1 \times$ PBS, and then harvested by pipetting for transfer into a 96-well plate. The spheroids were immersed in a $200 \mu \mathrm{L}$ volume of a $1: 1$ mixture of $1 \times \mathrm{PBS}$ and $0.1 \mathrm{M}$ sodium acetate buffer containing $0.1 \%$ Triton $\mathrm{X}-100$ and $400 \mu \mathrm{g} p$-nitrophenyl phosphate (Pierce Biotech Inc.) and incubated for 90 minutes at $37{ }^{\circ} \mathrm{C}$ in a $6 \% \mathrm{CO}_{2}$ atmosphere. Phosphatase activity was quenched by the addition of $10 \mu \mathrm{L}$ of $1 \mathrm{~N} \mathrm{NaOH}$ to each well and within 10 minutes the absorbance at $405 \mathrm{~nm}$ was measured using a plate reader (Multiskan ${ }^{\circledR}$ FC, Thermo Scientific). The experiment was undertaken in triplicate with each replicate involving the measurement of 8 spheroids.

\section{Results and discussion}

\section{Culture of uniformly sized spheroids on thin film PDMS arrays}

Spheroids were formed using a cell adhesion array prepared by thin film PDMS microcontact printing. ${ }^{13}$ PDMS surfaces resist cell adhesion and are well suited for confining cell patterns during prolonged culture. ${ }^{12,43}$ The patterned PDMS film was typically $40 \mathrm{~nm}$ thick, with perforations exposing the underlying tissue culture substrate to act as centres of cellular attachment for assembly and growth into spheroids. A hexagonal array was used throughout this study to provide equidistant spacing between spheroids and maintain near-uniform diffusion gradients 
surrounding the spheroids. An adhesion island diameter of $150 \mu \mathrm{m}$ was chosen for the culture of sufficiently large spheroids, while also being suitably small to enable the formation of spherical tissues and prevent the local formation of multiple, heterogeneously sized spheroids.

Traditional spheroid production methods involve compartmentalising cells within droplets or wells for the aggregation of the entire cell population over a period of 3-4 days. The array method partitions small sub-populations of proliferating cells within each adhesion island for growth into a compact cellular construct. Expansion from a small sub-population may more accurately reflect lineage expansion during in vivo tumour development. ${ }^{44}$ Assembly and growth of HT29 human colon carcinoma spheroids on arrays with a pitch of $450 \mu \mathrm{m}$ are documented in Fig. 1 and available as a time lapse video in the ESI $\dagger$. Cells were seeded for 24 hours followed by media exchange to remove non-adherent (non-viable) cells and reveal a well defined cellular array, with each adhesion island supporting 4050 cells (Fig. 1(A)). Following a further 24 hours of culture cell division produces a super-confluent monolayer (Fig. 1(B)). At this stage growth is constrained by the PDMS perimeter and the absence of further space for adhesion leads to the formation of a 3-D tissue, producing a hemispherical morphology by day 4 (Fig. 1(C)) and a spherical morphology with a diameter of $235 \mu \mathrm{m}(\mathrm{SD} \pm 12)$ by day 10 (Fig. 1(D)). Strong cell-cell and cell-

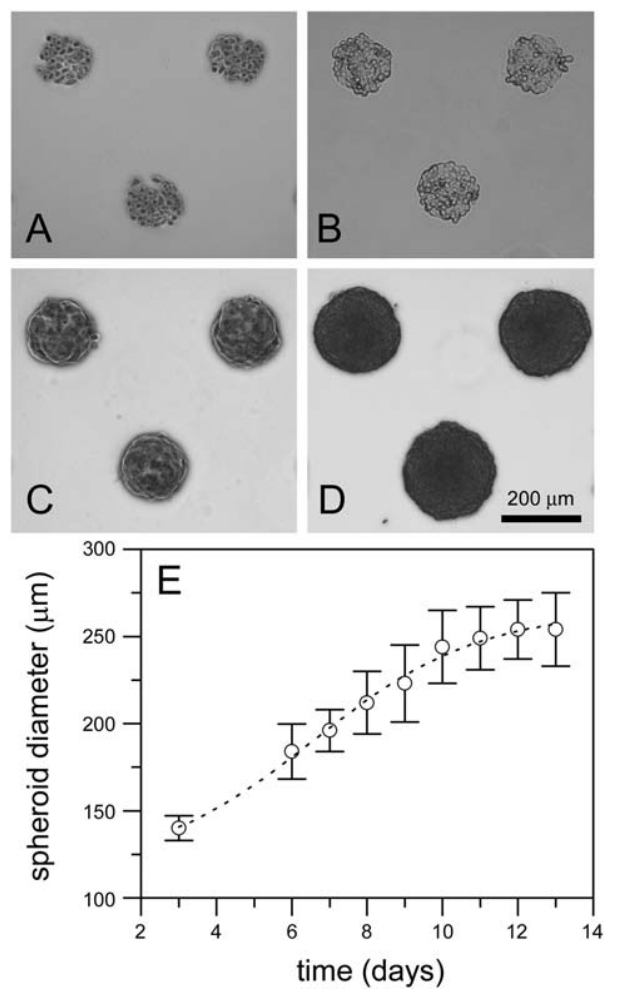

Fig. 1 Human HT29 colon carcinoma spheroid growth on arrays with a $450 \mu \mathrm{m}$ pitch: collections of cells adhered to the exposed areas of the glass substrate within the first day (A), monolayers were formed by day 2 (B), hemispheres were formed by day 4 (C) and plateau phase spheroids with a diameter $235 \mu \mathrm{m}(\mathrm{SD} \pm 12)$ were formed by day 10 (D). Spheroid growth during arrayed culture (E). A Boltzmann function was used to fit the sigmoidal curve. matrix interactions produce a collection of cells with a smooth surface. Indeed, during array-based culture E-cadherin gene expression is significantly up-regulated $(p<0.001)$ to promote cell-cell tethering and the formation of a tightly packed 3-D cellular body, whereas $\alpha 5 \beta 1$ integrin expression remains unaltered (see ESI, Fig. S2 $\dagger$ ). To demonstrate the wider applicability of the array method, spheroids were also formed from BT474 breast carcinoma and NCI-H1792 lung carcinoma cell lines (see ESI, Fig. S3†). Other spheroid-forming cell lines, including members of the NCI-DTP 60 -cell line screen, ${ }^{1,4}$ are also likely to be suitable for array-based culture.

Sigmoidal growth on the arrays into uniformly sized spheroids with diameters of $254 \mu \mathrm{m}(\mathrm{SD} \pm 21)$ is documented in Fig. 1(E). Shown in Fig. 2(A and B) the resulting tissues have a spherical morphology. The spheroids were harvested by pipetting and embedded in an agarose hydrogel for 3-axis imaging (see Fig. 2(C)). Circularity measurements of images from each axis were used to determine the truly spherical nature of the tissues (e.g. $x=91 \% ; y=93 \%$ and $z=94 \%$ ). Such sphericity is a prerequisite for a tissue model with radial internal mass transfer. The sphericity also enables the tissue density to be determined using sedimentation rate measurements.

\section{Mass production of tumour spheroids}

Arrays can be used as mother dishes for the highly parallel production of uniform tumour spheroids. The surface pattern partitions spheroid assembly and growth. This requires a single pipetting step to automate mass production, whereas individual pipetting operations are required for the formation of each spheroid by the hanging drop or the agarose overlay methods. The array method therefore eliminates pipetting errors and preparation is orders of magnitude faster. In addition, the array format anchors the spheroids in place for ease of media exchange without spheroid loss. The PDMS surface strongly resisted non-specific cell adhesion and the arrays had high across chip, chip-to-chip and batch-to-batch pattern occupancy levels $(>95 \%)$. To demonstrate the mass production capabilities, arrays with 1675 adhesion islands were each placed in a well of a standard 6-well culture plate (providing a total of 10050 adhesion islands) for spheroid culture (see Fig. 3). An average occupancy of $97.3 \%$ (9779 spheroids in total) resulted and, with a $99.0 \%$ harvesting efficiency, 9678 uniformly sized spheroids were obtained. Such levels of production ease requirements for incubator space and can match the demands of high throughput experimentation.

\section{Array pitch can be used to alter growth kinetics}

To determine the limits of spheroid growth an experiment using arrays with different pitches (from 400 to $2000 \mu \mathrm{m}$ ) was undertaken. The different growth curves are documented in Fig. 4(A). A pitch of $400 \mu \mathrm{m}$ produced spheroids with diameters of $200 \mu \mathrm{m}$ $(\mathrm{SD} \pm 11)$ by day 16 . Arrays with a $700 \mu$ m pitch produced HT29 spheroids with diameters of $276 \mu \mathrm{m}(\mathrm{SD} \pm 25)$ by day 17 , equivalent to HepG2 spheroids cultured on arrays with a $600 \mu \mathrm{m}$ pitch. ${ }^{34}$ Although these are the largest previously reported arraycultured spheroids, these are dwarfed by spheroids cultured on arrays with a $2000 \mu \mathrm{m}$ pitch. Here, rapid and sustained growth 

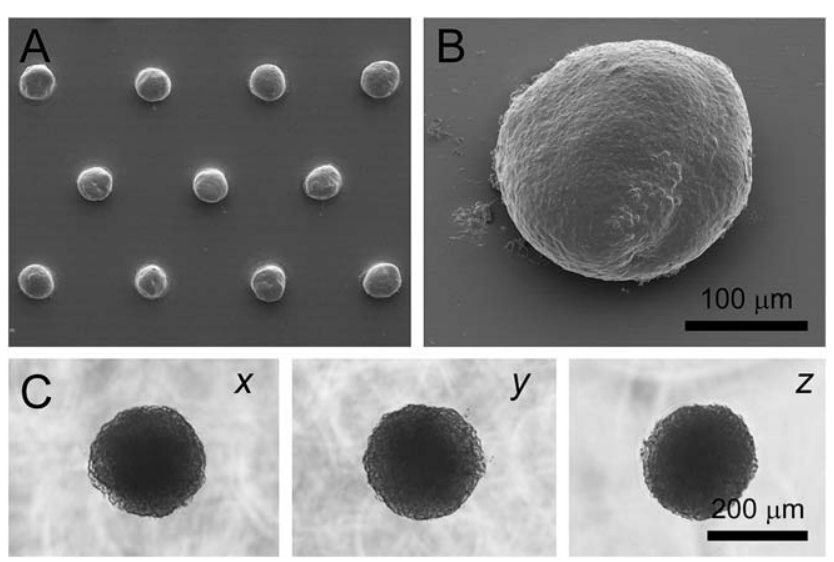

y

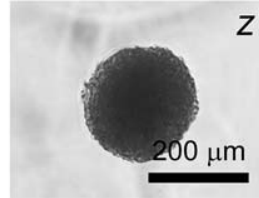

Fig. 2 SEM imaging of a formaldehyde-fixed array of uniformly shaped spheroids (A) and an individual adherent spheroid (B). Agarose embedding was used for 3-axis imaging $(C)$ and circularity measurements $(x=91 \% ; y=93 \%$; and $z=94 \%)$.

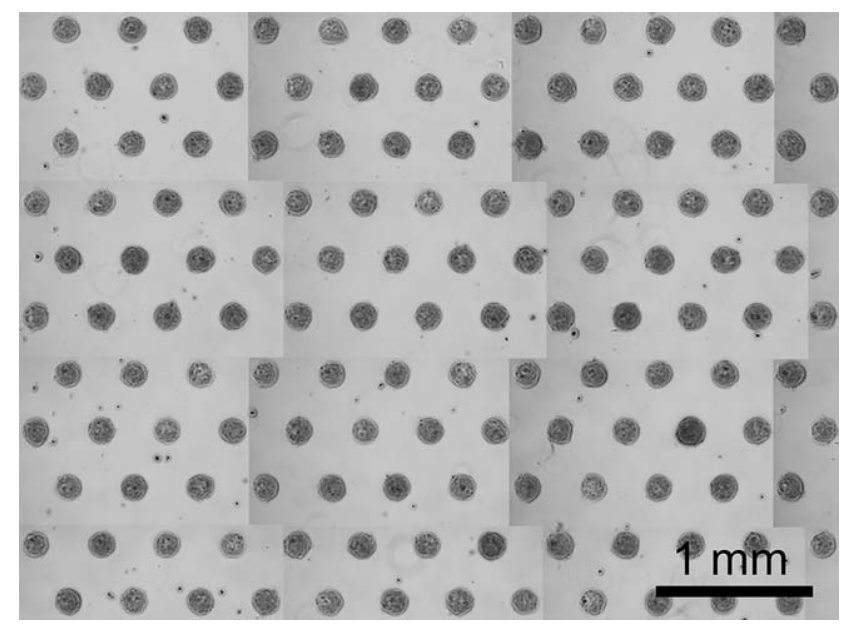

Fig. 3 Mass production of tumour spheroids on an array with a pitch of $450 \mu \mathrm{m}$. Image compilation of a $0.55 \mathrm{~cm}^{2}$ region of an array containing 137 uniformly sized spheroids following culture for 5 days.

enables the production of spheroids with diameters of $548 \mu \mathrm{m}$ $(\mathrm{SD} \pm 45)$ by day 27 . The sigmoidal growth characteristic indicates that, irrespective of the pitch, nutrient availability and waste removal ultimately become limiting during sustained culture.

To distinguish array pitch (area) from spheroid number (volume) factors, arrays with the same number (104) of adhesion islands and different pitches (from 400 to $2000 \mu \mathrm{m}$, Fig. 4(B)) were used, along with arrays with the same pitch $(400 \mu \mathrm{m})$ and different numbers of spheroids (from 104 to 2411, Fig. 4(C)). The number of adhesion islands per array had minimal impact on the growth, whereas the pitch of the arrayed adhesion islands dramatically affected growth (cell division rate), growth duration and consequently the final spheroid size. As the array density is reduced, from pitches of $400 \mu \mathrm{m}$ up to $1250 \mu \mathrm{m}$, the diffusive exchange of nutrients and waste becomes less limiting, leading to enhanced growth. Gains in growth are not observed beyond a pitch of $\geq 1250 \mu \mathrm{m}\left(\sim 1.35 \mathrm{~mm}^{2}\right.$ per spheroid $)$, the extent of the diffusive-competition for media. With these array dimensions the volume of available media instead becomes limiting. For example, the $2000 \mu \mathrm{m}$ pitch arrays provide $\sim 60 \mu \mathrm{L}$ of media per spheroid, a fraction of the $200 \mu \mathrm{L}$ used in the liquid overlay method. Under these conditions, the microarray format produces smaller plateau phase spheroids than the liquid overlay method which may in turn produce smaller spheroids than when using spinner flasks. Growth kinetics are therefore dictated by the chosen culture method and operating conditions. Growth on the microarray could be enhanced by the use of larger media volumes, more frequent media exchange (e.g. daily) or media perfusion. Without these considerations, the large pitch $(\geq 1250 \mu \mathrm{m})$ arrays still produce spheroids with sufficiently large $(\sim 500 \mu \mathrm{m})$ dimensions to impart in vivo-like mass transfer gradients and reflect the pathophysiological state of avascularised tumour regions.

Growth kinetics were also evaluated by curve fitting using the Gompertz equation, an established method for describing volumetric tumour spheroid growth. ${ }^{38}$ The reproducible morphology of spheroids cultured on the array (see Fig. 2) enabled the spheroid volume to be calculated using a capped spherical model. In the ESI Fig. S4†, volumetric growth is plotted with Gompertz curve fitting for spheroids cultured on arrays with pitches ranging from $400 \mu \mathrm{m}$ to $2000 \mu \mathrm{m}$. Curve fitting correlated well with the data $\left(r^{2} \geq 97 \%\right)$. The correlation increased with pitch to $r^{2} \geq 99 \%$ for the rapidly growing spheroids cultured on arrays with pitches $\geq 1500 \mu \mathrm{m}$, indicating that these arrays can be used to impart the same growth kinetics as other traditional spheroid culture methods.

Spheroids were cultured on arrays with different pitches and harvested at the plateau phase of growth for characterisation in terms of diameter, cell number, density and sphericity (see Table 1). The cell number was proportional to the spheroid diameter (and volume). The spheroids were characteristically dense, typically $1040 \mathrm{~kg} \mathrm{~m}^{-3}$, a density equivalent to those of in vivo tissues. This dense, robust character enables the spheroid to be harvested without damage for off-chip investigations. Circularity values from all 3 axes and all sizes were $\sim 90 \%$, indicating the formation of highly spherical tissue assemblies. Both the highly spherical morphology and the densely organized cellular structure are mandatory for an effective spheroid model. The reproducibility of these different characteristics underscores the value of array-based spheroid culture for the controlled formation of tumour spheroids with sizes ranging from 200$550 \mu \mathrm{m}$.

\section{Necrosis, proliferation and hypoxia marker profiles of plateau phase spheroids}

The spheroids were characterised in terms of proliferation, expression of hypoxia markers and necrosis. Hematoxylin and eosin stained median sections from plateau phase spheroids are shown in Fig. 5 and clearly reveal central secondary necroses for all three spheroid sizes (220 $\mu \mathrm{m}$ (A), $390 \mu \mathrm{m}$ (B) and $550 \mu \mathrm{m}(\mathrm{C}))$. The largest spheroids were further characterised. A BrdU incorporation assay was used to identify a narrow $(\sim 25 \mu \mathrm{m})$ proliferating layer of cells at the spheroid periphery (Fig. 5(D)), and immunostaining identified widespread expression of hypoxia inducible factor $1 \alpha$ (HIF-1 $\alpha)$ with high levels surrounding the 

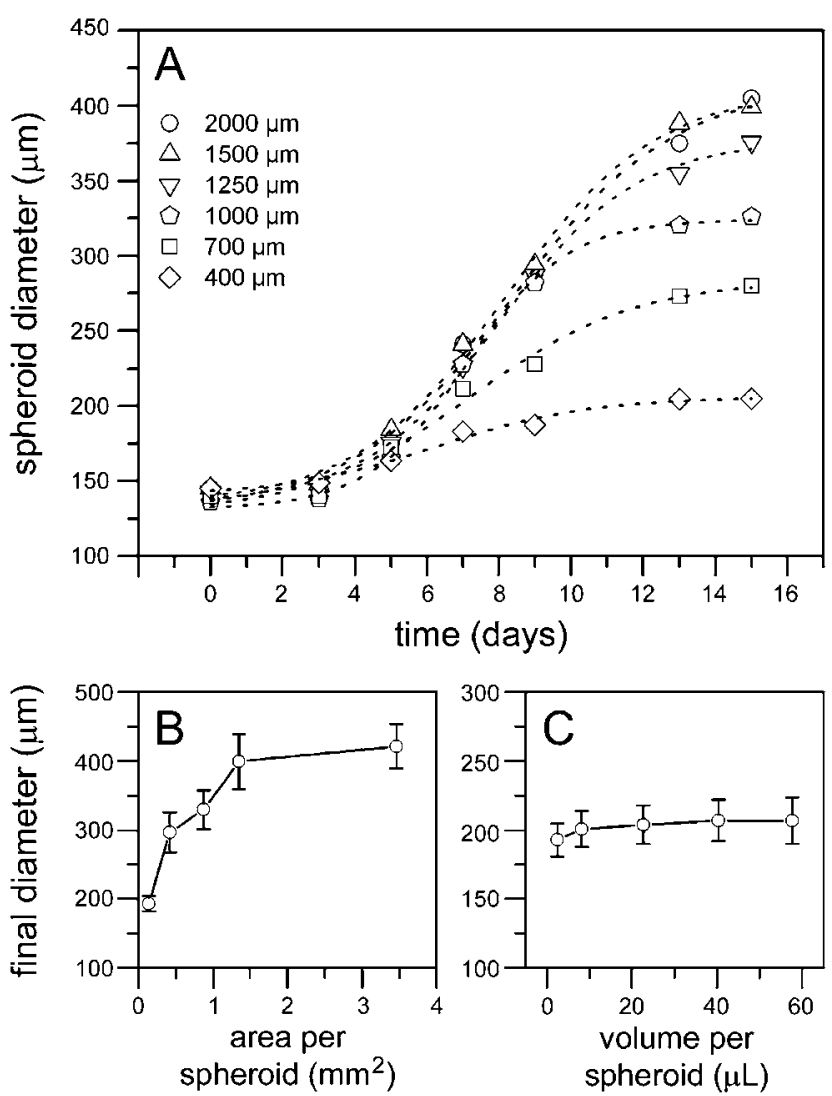

Fig. 4 Growth kinetics of spheroids cultured on arrays with pitches ranging from 400 to $2000 \mu \mathrm{m}$ (A). A Boltzmann function was used to fit the sigmoidal curves. Spheroid diameters were highly uniform $(\mathrm{SD} \leq$ $10 \%$ ). The available area per spheroid (array pitch: 400, 700, 1000, 1250 and $2000 \mu \mathrm{m}$ ) impacts final spheroid diameter (B). Each array contained 104 adhesion islands. With an array pitch of $400 \mu \mathrm{m}$, the media volume available per spheroid (spheroid number: 104, 351, 739, 1306 and 2411) had minimal effect on the final spheroid diameter (C). Data points are mean values from triplicate conditions, each involving 15-30 diameter measurements.

necrotic core (Fig. 5(E)). Combined, the different sections indicate that sustained culture on the arrays also produces diffusion gradients within the spheroid. The large spheroids formed on the array can therefore be used as models which reflect the pathophysiological state of naturally occurring avascularised microtumours or tumour microregions. The array approach also provides size tuning for the precise control of diffusion and thus metabolic gradients (i.e. the gradient is inversely proportional to the spheroid size). This degree of spatial control could be of particular value for investigations into the effects of a range of precisely defined biological gradients.

The expression of markers for proliferation, cell cycle arrest and hypoxia by plateau phase HT29 spheroids was further investigated by measuring gene expression levels of proliferating cell nuclear antigen (PCNA), cyclin D, p21 and vascularising endothelial growth factor (VEGF). Small $(\varnothing \approx 200 \mu \mathrm{m})$ and large $(\varnothing \approx 500 \mu \mathrm{m})$ spheroids were compared with monolayers cultured under hypoxic ( $1 \%$ oxygen) conditions as a control for a depleted oxygen state. All samples were referenced to monolayer cells cultured under normoxia conditions ( $20 \%$ oxygen). Documented in Fig. 6, both small and large spheroids have

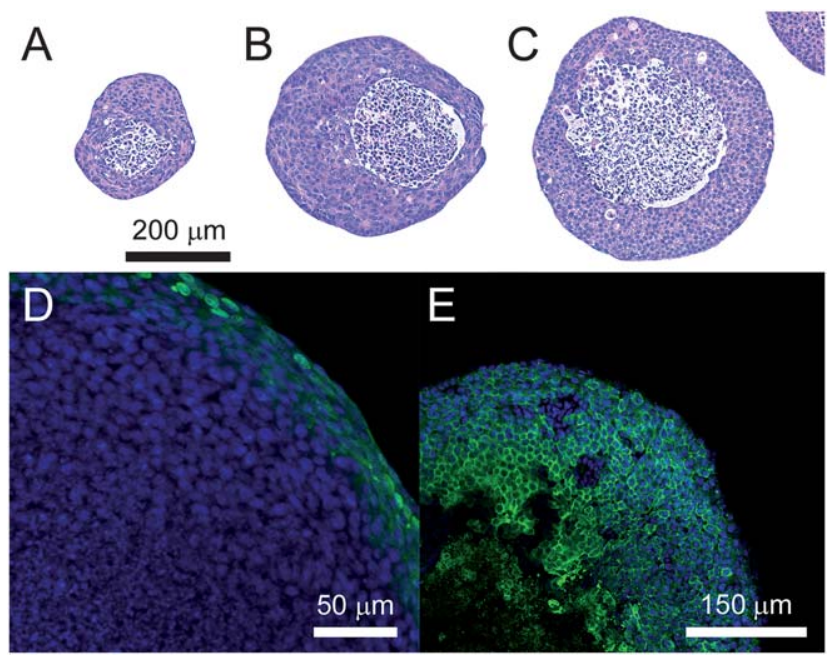

Fig. 5 Hematoxylin and eosin staining of median sections from a $220 \mu \mathrm{m}$ diameter spheroid (400 $\mu \mathrm{m}$ pitch; harvested day 16) (A), a $390 \mu \mathrm{m}$ diameter spheroid (1250 $\mu \mathrm{m}$ pitch; harvested day 17) (B) and a $550 \mu \mathrm{m}$ diameter spheroid (2000 $\mu \mathrm{m}$ pitch; harvested day 27) (C). BrdU incorporation (green) (D) and HIF-1 $\alpha$ (green) (E) distribution mapping by immunostaining of median sections from the largest spheroids. Nuclei were stained with DAPI (blue).

Table 1 Physical characteristics of tumour spheroids produced on arrays with different pitches. Spheroid mean diameter values are from 30 measurements, with the cell number obtained by triplicate pooling of 30 spheroids. Density values are the mean from 5 sedimentation rate measurements. The sphericity of the tumour spheroids was estimated by 3 -axis imaging of 5 spheroids followed by circularity measurements using ImageJ. The lowest circularity value was nominated as the $x$-axis value and the highest as the $z$-axis value. Three values of $100 \%$ indicate perfect sphericity. Standard deviation values are prefixed with \pm

\begin{tabular}{|c|c|c|c|c|c|c|c|}
\hline Array pitch/ $\mu \mathrm{m}$ & Harvested/day & Diameter/ $\mu \mathrm{m}$ & Cell number & Density $/ \mathrm{kg} \mathrm{m}^{-3}$ & \multicolumn{3}{|c|}{ Circularity $(\%)$} \\
\hline 700 & 17 & $276 \pm 25$ & $5984 \pm 297$ & $1047 \pm 6$ & $89 \pm 3$ & $91 \pm 3$ & $94 \pm 3$ \\
\hline 1000 & 17 & $324 \pm 30$ & $9438 \pm 955$ & $1048 \pm 5$ & $90 \pm 2$ & $91 \pm 2$ & $94 \pm 2$ \\
\hline 1250 & 17 & $379 \pm 38$ & $15676 \pm 709$ & $1037 \pm 5$ & $89 \pm 3$ & $92 \pm 2$ & $94 \pm 3$ \\
\hline 1500 & 27 & $531 \pm 54$ & - & $1033 \pm 5$ & $89 \pm 2$ & $91 \pm 2$ & $92 \pm 2$ \\
\hline
\end{tabular}


expression patterns similar to those from monolayers cultured under hypoxic conditions. Down regulation of PCNA and cyclin $\mathrm{D}$ along with a marked increase in p21 levels indicate cell cycle arrest. VEGF gene expression, a classic marker for hypoxic stress (and relevant to a variety of tumour cells), was equally upregulated in the small (4.23-fold, $\mathrm{SD} \pm 1.40$ ) and large (4.28-fold, $\mathrm{SD} \pm 0.33)$ spheroids, and naturally strongly up-regulated in the hypoxic monolayers (17.14-fold, $\mathrm{SD} \pm 2.67$ ). These data indicate that both small and large spheroids experience hypoxia. In addition to oxygen starvation other metabolic gradients and pathophysiological states will also result during sustained culture.

\section{Onset of necrosis and hypoxia}

An experiment involving hematoxylin and eosin staining of median spheroid sections was used to determine the onset of secondary necrosis in spheroids cultured on $400 \mu \mathrm{m}$ pitch and $1500 \mu \mathrm{m}$ pitch arrays. As shown in ESI Fig. S5(A)†, on arrays with a $400 \mu \mathrm{m}$ pitch secondary necrosis became evident $(<5 \%$ by volume) by day 14 with spheroid diameters of $202 \mu \mathrm{m}(\mathrm{SD} \pm 13)$. On the $1500 \mu \mathrm{m}$ pitch arrays, secondary necrosis also became evident ( $<5 \%$ by volume) by day 14 in spheroids with diameters of $389 \mu \mathrm{m}(\mathrm{SD} \pm 26)$. This conflicts with observations that necrosis onset is determined by scale, occurring within 100 to $200 \mu \mathrm{m}$ diameter spheroids. ${ }^{34,45}$ However, these investigations used microporous or microarray systems with pitches far beneath the $\sim 1250 \mu \mathrm{m}$ distance required for optimal array-based growth (see Fig. 4(B)). These results point to the importance of both scale and the culture history in the development of metabolic gradients and secondary necrosis, and highlight the critical importance of low microwell and microarray densities for the sustained culture of large and viable spheroids.
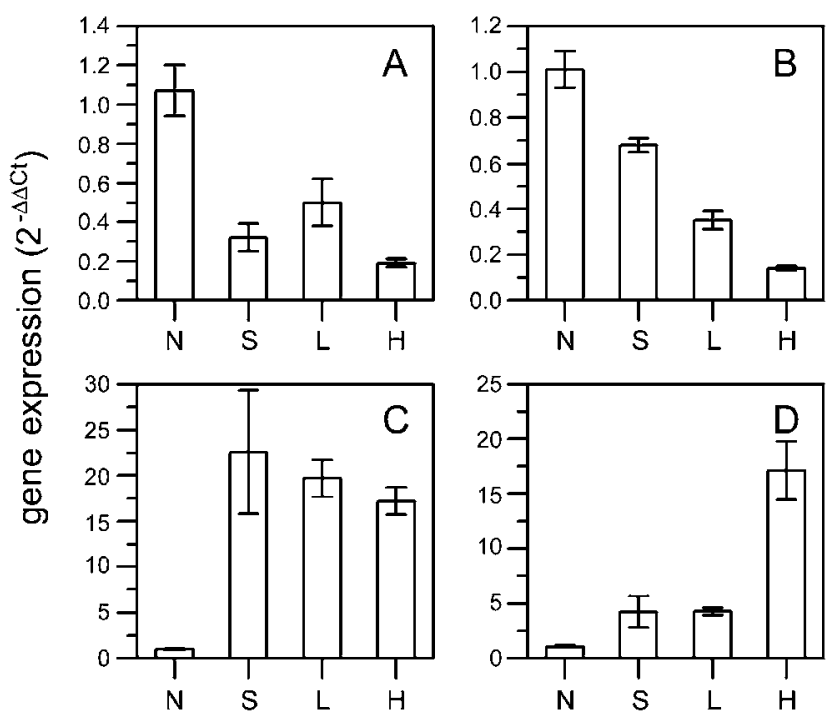

Fig. 6 PCNA (A), cyclin D (B), p21 (C) and VEGF (D) gene expression levels from small $(S: \sim 200 \mu \mathrm{m}$ diameter, day 14) and large $(L: \sim 500 \mu \mathrm{m}$ diameter, day 21) plateau phase spheroids were compared with levels from hypoxic $(H)$ monolayer cultures with $1 \% \mathrm{O}_{2}$ levels. Values are normalised relative to normoxia $(N, 20 \%$ oxygen) monolayer cultures. Data points are mean expression values \pm standard deviation from triplicate conditions, with triplicate measurements from each sample.
Secondary necrosis is a late stage response to nutrient deprivation, with oxygen availability being one of many factors affecting cell metabolism and viability. To gain insight into the earlier onset of hypoxic stress within the spheroids VEGF gene expression was used as a diagnostic marker. Results are plotted with diameter values in Fig. 7. Expression levels from spheroids cultured on the dense $400 \mu \mathrm{m}$ pitch arrays were significantly $(p<0.001)$ up-regulated by day 7 with diameters of $179 \mu \mathrm{m}$ $(\mathrm{SD} \pm 14)$. On the $1500 \mu \mathrm{m}$ pitch arrays, VEGF expression became significantly $(p<0.001)$ up-regulated by day 10 with diameters of $320 \mu \mathrm{m}(\mathrm{SD} \pm 29)$. The later response shows the enhanced oxygen supply for rapid growth provided by the $1500 \mu \mathrm{m}$ pitch arrays. Importantly, these results demonstrate that modulation of the array density can be used to produce well defined spatio-temporal models of developing pathophysiology. Furthermore, the identification of pre-hypoxic and hypoxic (but pre-necrotic) culture phases can be used to define windows of operation for experiments aiming to elucidate the effect of metabolic status on the responsiveness of spheroids to anticancer treatments.

For applications requiring fully viable spheroids the microarray format can serve as a mother dish, like the hanging drop method, with spheroid harvesting prior to the onset of metabolic stress and pathophysiology. The robust, densely aggregated character of the spheroids enables the harvesting of intact spheroids by pipetting. However, spheroids harvested from arrays with a $400 \mu \mathrm{m}$ pitch at an early stage (day 7) of maturation have a capped spherical morphology, requiring a further 24 hours culture within a non-adherent culture flask for natural shape remodelling ${ }^{46}$ into a highly spherical tissue $(x=91 \%$; $y=95 \%$; and $z=96 \%$ ).

\section{Spheroids have significantly reduced chemosensitivity}

Chemotherapy investigations can be undertaken directly on the array. We have demonstrated this using an in situ dose-response experiment with the anti-cancer agent irinotecan. Arrays with pitches of $400 \mu \mathrm{m}$ and $1500 \mu \mathrm{m}$ were selected to compare the responses of rapid and slow growing spheroids. In addition, prehypoxic and hypoxic (but pre-necrotic) phases of culture were compared. The experiments entailed irinotecan exposure for 3 days followed by measuring the acid phosphatase activity, a reliable indicator of the viable cell count of HT29 spheroids with diameters as large as $650 \mu \mathrm{m} .{ }^{42}$

An irinotecan dose-response experiment involving monolayer cultures gave a $50 \%$ inhibition concentration $\left(\mathrm{IC}_{50}\right)$ value of $32 \mu \mathrm{M}(95 \%$ CI $22-49)$, significantly $(p<0.001)$ lower than the spheroid culture $\mathrm{IC}_{50}$ values. Documented in Fig. 8(A), prehypoxic phase spheroids cultured on the $400 \mu \mathrm{m}$ pitch arrays had an $\mathrm{IC}_{50}$ of $102 \mu \mathrm{M}(95 \% \mathrm{CI} 49-239)$ which was significantly ( $p<$ $0.001)$ increased to $307 \mu \mathrm{M}(95 \%$ CI 144-634) during the hypoxic culture phase (see Fig. 8(A)). The pre-hypoxic phase spheroids cultured on the $1500 \mu \mathrm{m}$ pitch arrays had an $\mathrm{IC}_{50}$ of $62 \mu \mathrm{M}(95 \%$ CI 23-96) which was also significantly $(p<0.001)$ increased to $224 \mu \mathrm{M}(95 \%$ CI 123-408) in the hypoxic culture phase (see Fig. 8(B)). The culture conditions, treatment periods, spheroid diameters, volumetric growth and $\mathrm{IC}_{50}$ values are summarised in the ESI, Table $\mathrm{S} 1 \dagger$. Together the results demonstrate that 

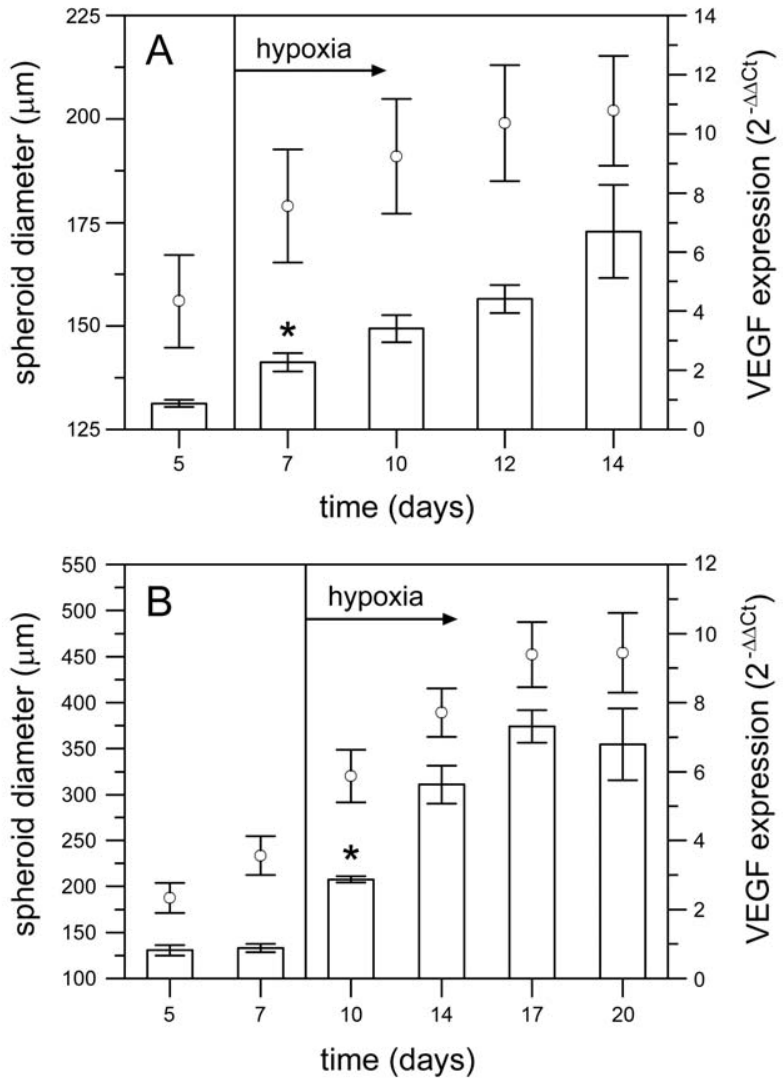

Fig. 7 Diameter (circles) and VEGF gene expression (bars) during spheroid culture for up to 20 days on arrays with pitches of $400 \mu \mathrm{m}$ (A) and $1500 \mu \mathrm{m}$ (B). Mean diameter values \pm standard deviation are from 30 measurements. Expression levels are normalised relative to levels from monolayer cultures. Data points are mean expression values \pm standard deviation from triplicate conditions, with triplicate measurements from each sample $(* p<0.001)$.

array-cultured spheroids have markedly reduced sensitivity towards irinotecan therapy than simple monolayer cultures.

The 3-D character, scale, cell proliferation rate and hypoxia state are all factors which can lead to reduced drug efficacy. The anti-tumour activity of irinotecan involves killing cells in S-phase by inhibiting DNA synthesis. ${ }^{47,48}$ This mechanism explains the reduced efficacy during treatment of the slowly proliferating spheroids cultured on the $400 \mu \mathrm{m}$ pitch arrays. As revealed by the diameter and volumetric growth data (see ESI, Table S1†), the rate of cell proliferation tends to zero (i.e. total cell cycle arrest) in the hypoxic phase of culture, producing spheroids that are only marginally larger (by $17 \mu \mathrm{m}$ ) than those cultured in the prehypoxic culture phase. This loss of proliferation (without an appreciable increase in size) acts to further curb the efficacy of irinotecan $\left(\mathrm{IC}_{50}=307 \mu \mathrm{M}\right)$. In comparison, the spheroids cultured on the $1500 \mu \mathrm{m}$ pitch arrays have high proliferation rates throughout the experimental time scales (see ESI, Table $\mathrm{S} 1 \dagger$ ), producing $240 \mu \mathrm{m}$ diameter spheroids in the measured prehypoxic growth phase and $363 \mu \mathrm{m}$ diameter spheroids in the measured hypoxic growth phase. Here the reduced chemosensitivity may to be attributed to the limited penetration of irinotecan into these large tissues. However, in the hypoxic phase of culture it is uncertain whether the increased scale (by $123 \mu \mathrm{m}$ )
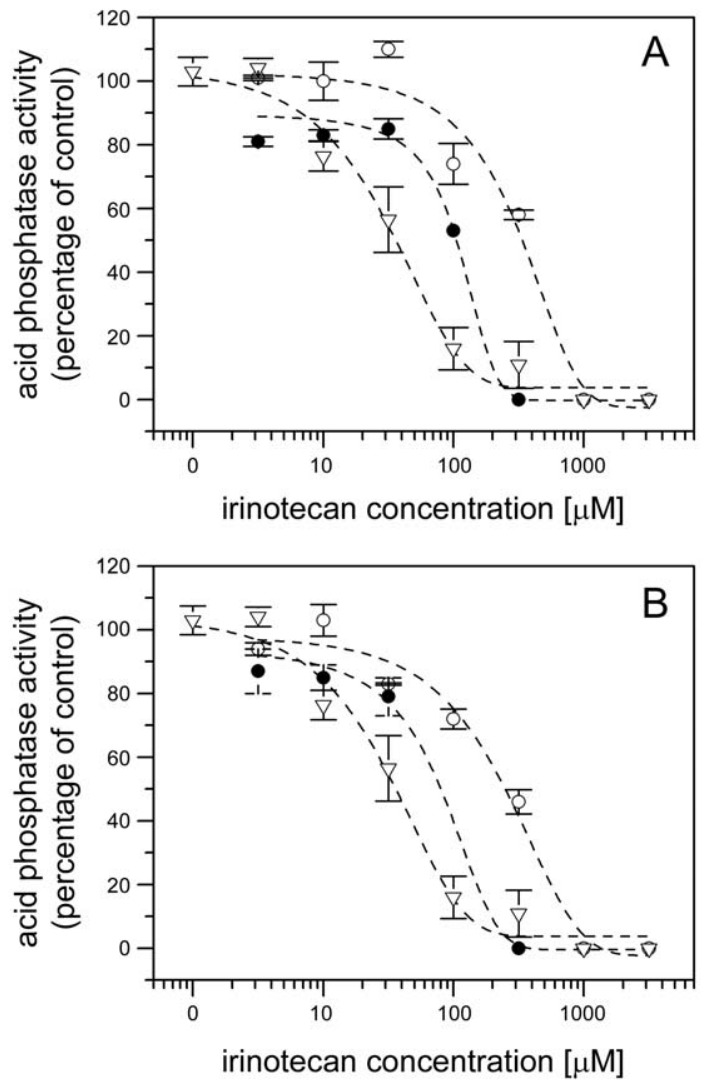

Fig. 8 Irinotecan dose-response results for spheroids cultured on $400 \mu \mathrm{m}$ pitch (A) and $1500 \mu \mathrm{m}$ pitch (B) arrays. The irinotecan concentration is plotted against acid phosphatase activity (relative to untreated controls) for monolayer cultures (triangles), pre-hypoxic spheroids (filled circles) and hypoxic spheroids (white circles) following 3 days of exposure to irinotecan. Curves were fitted using a Hill function with variable slope. Mean values \pm standard deviation are from triplicate experiments, with each replicate involving the measurement of 8 individual spheroids.

and/or the altered metabolic state causally relate to the reduced drug efficacy $\left(\mathrm{IC}_{50}=224 \mu \mathrm{M}\right)$. Further experiments using the microarray format are required to accurately discern the relative contributions of the different factors.

\section{Future perspectives}

The presented research has focussed on growth, scale and hypoxia factors that affect sensitivity to treatment. A more comprehensive understanding can be obtained by the quantitative and spatial characterisation of other developing metabolic transitions. In addition to chemosensitivity experiments, the spheroid microarray format can be used for the evaluation of radio- and immunotherapies, for the formation of more complex spheroid co-cultures and for addressing fundamental lines of research in cancer biology. ${ }^{2}$

The array format also has the potential to be used for growth inhibition experiments with assay coordinates provided for in situ monitoring. To clearly discern inhibitory effects, large pitch arrays will be required to support optimal growth. This method can be combined with automated imaging for a high content 
screening approach to therapy testing. The in situ anti-cancer therapy screening methods can be further improved by packaging the spheroid arrays within the industry standard microtitre plate format for lower volume drug testing. The PDMS material used for cell patterning is also ideally suited for plasma bonding ${ }^{49}$ approaches to packaging. Each well of a 96 -well plate $(\varnothing=$ $6.5 \mathrm{~mm}$ ) can accommodate 14 spheroids arrayed with a pitch of $1500 \mu \mathrm{m}$ to provide more replicates for enhanced statistical confidence, or 3 spheroids within each well of a 384-well plate $(\varnothing=3.0 \mathrm{~mm})$ for higher throughput screening. Dense arrays, with a $400 \mu \mathrm{m}$ pitch, can be used to produce large numbers of small spheroids; 480 within each well of a 96-well plate or 100 within each well of a 384-well plate. Such simple, yet powerful, developments have the potential to greatly enhance the value of the cell array format for spheroid research in both academic and industrial settings.

\section{Conclusions}

Microarrays can be used for the inherently automated and scalable production of uniform tumour spheroids with diameters as large as $550 \mu \mathrm{m}$. The array density dictates the growth dynamics, with optimal growth kinetics on arrays with a pitch of $\geq 1250 \mu \mathrm{m}$. The array format produces mass transfer gradients surrounding and within the spheroids. Coupled with precision size control array-based culture can be used to produce spatiotemporal tissue models for understanding the development of metabolic gradients and pathophysiological states. Fully viable spheroids can be readily harvested or remain in situ and used to investigate the impact of scale, cell proliferation rate and metabolic status on the responsiveness to candidate anti-cancer therapies. Together these developments portray the large potential of the array format to support the widespread adoption of the tumour spheroid model, provide detailed insights into responses to pathophysiological gradients and aid the high information content screening of new anti-cancer treatment strategies.

\section{Acknowledgements}

The authors are indebted to Ulrich Marggraf (TU Dortmund, MST) for SU-8 master fabrication, Maria Becker (ISAS) for SEM analysis, Melanie Huether (OncoRay) and Brigitte BegherTibbe (IfADo) for sectioning and staining, Julia Sisnaiske (IfADo) for time lapse microscopy, Christoph van Thriel (IfADo) for support with statistics, and Sarah Waide and Susanne Funken (ISAS) for cell culture support. The interdisciplinary research was financially supported by the German Research Foundation (DFG grants WE3737/3-1 and KU971/7-1), the BMBF (NGFN project OncoProfile) and the Ministry of Innovation, Science, Research and Technology of the state of North Rhine-Westphalia. Heike Hardelauf also thanks the International Leibniz Graduate School "Systems Biology Lab-ona-Chip" for financial support.

\section{References}

1 J. Friedrich, R. Ebner and L. A. Kunz-Schughart, Int. J. Radiat. Biol., 2007, 83(11-12), 849-871.
2 F. Hirschhaeuser, H. Menne, C. Dittfeld, J. West, W. Mueller-Klieser and L. A. Kunz-Schughart, J. Biotechnol., 2010, 148(1), 3-15.

3 M. Ingham, G. B. Techy, R. Saroufeem, O. Yazan, K. S. Narayan, T. J. Goodwin and G. F. Spaulding, In Vitro Cell. Dev. Biol.: Anim., 1997, 33, 459-466.

4 J. Friedrich, C. Seidel, R. Ebner and L. A. Kunz-Schughart, Nat. Protoc., 2009, 4(11-12), 309-324.

5 A. Ivascu and M. Kubbies, J. Biomol. Screening, 2006, 11, 922-932.

6 R. M. Sutherland, Science, 1988, 240, 177-184.

7 J. M. Kelm and M. Fussenegger, Trends Biotechnol., 2004, 22(4), 195202.

8 N. E. Timmins and L. K. Nielsen, Methods Mol. Med., 2007, 140, $141-151$.

9 X. Zhang, W. Wang, W. Yu, Y. Xie, X. Zhang, Y. Zhang and X. Ma, Biotechnol. Prog., 2005, 21(4), 1289-1296.

10 J. Lee, M. J. Cuddihy, G. M. Cater and N. A. Kotov, Biomaterials, 2009, 30, 4687-4694.

11 S. Sakai, S. Ito, Y. Ogushi, I. Hashimoto, N. Hosoda, Y. Sawae and K. Kawakami, Biomaterials, 2009, 30(30), 5937-5942.

12 J.-P. Frimat, H. Menne, A. Michels, S. Kittel, R. Kettler, S. Borgmann, J. Franzke and J. West, Anal. Bioanal. Chem., 2009, 395(3), 601-609.

13 J.-P. Frimat, J. Sisnaiske, S. Subbiah, H. Menne, P. Godoy, P. Lampen, M. Leist, J. Franzke, J. G. Hengstler, C. van Thriel and J. West, Lab Chip, 2010, 10, 701-709.

14 M. J. Powers, K. Domansky, M. R. Kaazempur-Mofrad, A. Kalezi, A. Capitano, A. Upadhyaya, P. Kurzawski, K. E. Wack, D. B. Stolz, R. Kamm and L. G. Griffith, Biotechnol. Bioeng., 2002, 78, 257-269.

15 Y. Torisawa, B. Chueh, D. Huh, P. Ramamurthy, T. M. Roth, K. F. Barald and S. Takayama, Lab Chip, 2007, 7, 770-776.

16 Y. Torisawa, B. Mosadegh, G. D. Luker, M. Morell, K. S. O’Shea and S. Takayama, Integr. Biol., 2009, 1, 649-654.

17 A. Y. Hsiao, Y. Torisawa, Y.-C. Tung, S. Sud, R. S. Taichman, K. J. Pienta and S. Takayama, Biomaterials, 2009, 30, 3020-3027.

18 K. Kamei, S. Guo, Z. T. F. Yu, H. Takahashi, E. Gschweng, C. Suh, X. Wang, J. Tang, J. McLaughlin, O. N. Witte, K.-B. Lee and H.-R. Tseng, Lab Chip, 2009, 9, 553-563.

19 Y. C. Toh, C. Zhang, J. Zhang, Y. M. Khong, S. Chang, V. D. Samper, D. van Noort, D. W. Hutmacher and H. Yu, Lab Chip, 2007, 7, 302-309.

20 S.-W. Ong, C. Zhang, Y. C. Toh, S. H. Kim, H. L. Foo, C. H. Tan, D. van Noort, S. Park and H. Yu, Biomaterials, 2008, 29, 3237-3244.

21 L. Y. Wu, D. Di Carlo and L. P. Lee, Biomed. Microdevices, 2008, 10, 197-202.

22 A. Iwasaki, T. Matsumoto, G. Tazaki, H. Tsuruta, H. Egusa, H. Miyajima and T. Sohmura, Adv. Eng. Mater., 2009, 11(10), 801804.

23 M. D. Ungrin, C. Joshi, A. Nica, C. Bauwens and P. W. Zandstra, PLoS One, 2008, 3(2), e1565.

24 K. Nakazawa, Y. Izumi, J. Fukuda and T. Yasuda, J. Biomater. Sci., Polym. Ed., 2006, 17(8), 859-873.

25 J. Park, C. H. Cho, N. Parashurama, Y. Li, F. Berthiaume, M. Toner, A. W. Tilles and M. L. Yarmush, Lab Chip, 2007, 7, 1018-1028.

26 D. Gallego-Perez, N. Higuita-Castro, S. Sharma, R. K. Reen, A. F. Palmer, K. J. Gooch, L. J. Lee, J. J. Lannutti and D. J. Hansford, Lab Chip, 2010, 10, 775-782.

27 J. M. Karp, J. Yeh, G. Eng, J. Fukuda, J. Blumling, K.-Y. Suh, J. Cheng, A. Mahdavi, J. Borenstein, R. Langer and A. Khademhosseini, Lab Chip, 2007, 7, 786-794.

28 Y. Sakai and K. Nakazawa, Acta Biomater., 2007, 3, 1033-1040.

29 J. Fukuda, Y. Sakai and K. Nakazawa, Biomaterials, 2006, 27, 10611070.

30 R. Mori, Y. Sakai and K. Nakazawa, J. Biosci. Bioeng., 2008, 106(3), 237-242.

31 R. Inaba, A. Khademhosseini, H. Suzuki and J. Fukuda, Biomaterials, 2009, 30, 3573-3579.

32 H. Otsuka, A. Hirano, Y. Nagasaki, T. Okano, Y. Horiike and K. Kataoka, ChemBioChem, 2004, 5, 850-855.

33 W. Wang, K. Itaka, S. Ohba, N. Nishiyama, U. Chung, Y. Yamasaki and K. Katoaka, Biomaterials, 2009, 30, 2705-2715.

34 T. Tamura, Y. Sakai and K. Nakazawa, J. Mater. Sci.: Mater. Med., 2008, 19, 2071-2077.

35 K. Yoshimoto, M. Ichino and Y. Nagasaki, Lab Chip, 2009, 9, $1286-$ 1289. 
36 R. Kojima, K. Yoshimoto, E. Takahashi, M. Ichino, H. Miyoshi and Y. Nagasaki, Lab Chip, 2009, 9, 1991-1993.

37 G. F. Brunton and T. E. Wheldon, Cell Tissue Kinet., 1980, 13, 455460.

38 L. A. Kunz-Schughart, K. Groebe and W. Mueller-Klieser, Int. J. Cancer, 1996, 66, 578-586.

39 R. Chignola, A. Schenetti, G. Andrighetto, E. Chiesa, R. Foroni, S. Sartoris, G. Tridente and D. Liberati, Cell Proliferation, 2000, 33(4), 219-229.

40 K. J. Livak and T. D. Schmittgen, Methods, 2001, 25(4), 402-408.

41 T. T. Yang, P. Sinai and S. R. Kain, Anal. Biochem., 1996, 241, 103 108.

42 J. Friedrich, W. Eder, J. Castaneda, M. Doss, E. Huber, R. Ebner and L. A. Kunz-Schughart, J. Biomol. Screening, 2007, 12(7), 925-937.
43 M. N. De Silva, R. Desai and D. J. Odde, Biomed. Microdevices, 2004, 6, 219-222.

44 M. F. Clarke, J. E. Dick, P. B. Dirks, C. J. Eaves, C. H. M. Jamieson, D. L. Jones, J. Visvader, I. L. Weissman and G. M. Wahl, Cancer Res., 2006, 66, 9339-9344.

45 R. Glicklis, J. C. Merchuk and S. Cohen, Biotechnol. Bioeng., 2004, 86(6), 672-680.

46 N. C. Rivron, PhD. thesis, Universiteit Twente, 2010

47 C. Jaxel, K. W. Kohn, M. C. Wani, M. E. Wall and Y. Pommier, Cancer Res., 1989, 49(6), 1465-1469.

48 Y.-H. Hsiang, M. G. Lihou and L. F. Liu, Cancer Res., 1989, 49(18), 5077-5082.

49 M. K. Chaudhury and G. M. Whitesides, Science, 1992, 255, 12301232. 